This paper is a postprint of a paper submitted to and accepted for publication in IET Microwaves,

Antennas \& Propagation and is subject to Institution of Engineering and Technology Copyright.

The copy of record is available at IET Digital Library

IET Microwaves, Antennas \& Propagation

\title{
Efficient modal analysis of arbitrarily shaped $H$-plane two-port waveguide devices using the 2D parallel-plate Green's function
}

\author{
A. Belenguer ${ }^{1}$ H. Esteban ${ }^{2}$ V.E. Boria ${ }^{2}$ J.V. Morro ${ }^{2}$ \\ C. Bachiller ${ }^{2}$
}

${ }^{1}$ Departamento de Ingenieria Electrica, Electronica, Automatica y Comunicaciones, E.U. Politécnica, Universidad de Castilla-La Mancha, Campus Universitario, 16071 Cuenca, Spain

${ }^{2}$ Departamento de Comunicaciones, Universidad Politécnica de Valencia, Camino de Vera, s/n, 46022 Valencia, Spain E-mail: hesteban@dcom.upv.es

\begin{abstract}
A very efficient method for the accurate analysis of arbitrarily shaped $\mathrm{H}$-plane two-port structures in rectangular waveguide technology is presented. The method is based on the integral-equation technique, which makes use of the Green's function of an infinite line source placed inside two parallel plates. All the terms of the series of the Green's function are analytically integrated, thus resulting in new series with accelerated convergence. After performing an exhaustive convergence analysis, optimal values for the parameters of this new method have been found and used in all calculations. The new method has been successfully tested after analysing several practical $\mathrm{H}$-plane structures, such as inductive filters including the mechanisation effect of rounded corners.
\end{abstract}

\section{Introduction}

The integral-equation (IE) technique has been widely applied to the accurate analysis of antennas and scattering problems in free-space $[1,2]$. In order to solve the integral equations arising in such problems, where no boundary conditions are imposed to the required Green's function, the well-known method of moments (MoM) is typically employed [3]. This set of numerical techniques can also be successfully applied to electromagnetic problems with boundary conditions, such as the full-wave analysis of $\mathrm{H}$-plane problems in waveguide technology [4-6]. This kind of problems are of great practical interest, since many rectangular waveguide devices based on inductive metal obstacles are widely used in telecommunication applications, as it can be inferred from several recent publications [7-11].

In order to solve $\mathrm{H}$-plane rectangular waveguide problems, it is necessary to make use of an appropriate Green's function including the corresponding metal boundary conditions, which represents the scattering of an infinite line source between two parallel plates [12]. The Green's function for a parallel-plate waveguide (PPW), defined as the summation of a series of guided modes [13], has been previously used to solve the scattering of a single inductive post in a rectangular waveguide $[14,15]$. Other IE techniques have also made use of such Green's function for the analysis of an arbitrary number of $2 \mathrm{D}$ metallic posts placed inside a rectangular waveguide [16, 17]. However, the proposed PPW Green's function consists of slowly converging series, whose convergence rate has been accelerated either using the Kummer's transformation [14, 17], or employing particular basis functions in the application of MoM [16]. Nevertheless, the kind of inductive geometries that can be successfully considered following such techniques is restricted.

In this paper, we propose an alternative solution for improving the accuracy and numerical efficiency related to the modal analysis of arbitrarily shaped $\mathrm{H}$-plane two-port waveguide devices using the PPW Green's function. Instead of directly evaluating the cited Green's function in 
series form, we propose to evaluate first the integrals related to the application of the MoM-based IE technique considering spatially distributed currents, and then proceed with the summation of the resulting terms. Proceeding in this way, we prove that the convergence rate of this new algorithm is increased with regard to any other previous classical solution. Furthermore, the use of distributed sources also allows to place them along the metallic contour of the scatterer, thus greatly improving the accuracy related to this new analysis technique. For validating all the advantages related to this new technique, we have successfully applied it to the full-wave analysis of several inductively coupled rectangular waveguide filters, even considering typical rounded corner mechanisation effects related to milling manufacturing techniques.

\section{Problem formulation}

The aim of this work is the analysis of H-plane structures in rectangular waveguide technology. These structures, when fed with the fundamental mode $T E_{10}$, only excite $T E_{n 0}$ modes [18]. If we use the coordinate system of Fig. 1, this means that only the $H_{x}, H_{y}$ and $E_{z}$ components are nonzero. Thus, the analysis can be reduced to a $2 \mathrm{D} z$-invariant scattering problem with $T M^{z}$ incidence, and it is not necessary to consider the upper and lower walls of the guide.

The incident $\left(E_{z}^{\mathrm{i}}\right)$ and scattered fields $\left(E_{z}^{\mathrm{s}}\right)$ can be related through [1]

$$
E_{z}^{\mathrm{s}}(x, y)=\jmath k \eta \int_{T} J_{z}\left(x^{\prime}, y^{\prime}\right) G\left(x, y ; x^{\prime}, y^{\prime}\right) \mathrm{d} t^{\prime}
$$

where $T$ stands for the scatterer contour, $k$ the wave number, $\eta$ the medium impedance, $(x, y)$ the location of the field point, $\left(x^{\prime}, y^{\prime}\right)=\left(x^{\prime}\left(t^{\prime}\right), y^{\prime}\left(t^{\prime}\right)\right)$ the source point, and $G$ the PPW Green's function.

The PPW Green's function represents the scattering of a line source placed at $\left(x^{\prime}, y^{\prime}\right)$ between two parallel plates. Thus, it must accomplish the following boundary conditions

$$
G\left(x, 0, x^{\prime}, y^{\prime}\right)=0 \quad G\left(x, a, x^{\prime}, y^{\prime}\right)=0
$$

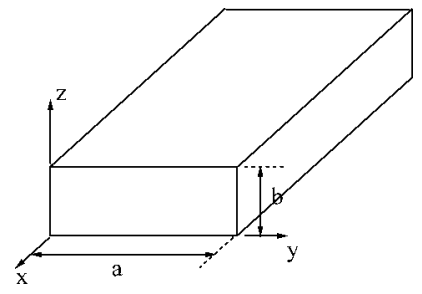

Figure 1 Coordinate system used in the rectangular waveguide and the following wave equation

$\nabla^{2} G\left(x, y ; x^{\prime}, y^{\prime}\right)+k^{2} G\left(x, y ; x^{\prime}, y^{\prime}\right)=\delta\left(x-x^{\prime}\right) \delta\left(y-y^{\prime}\right)$

It is possible to obtain an analytical expression for $G$ in series form [19]

$$
\begin{aligned}
G\left(x, y ; x^{\prime}, y^{\prime}\right)= & \frac{j}{a} \sum_{m=1}^{\infty} \frac{\sin ((m \pi / a) y) \sin \left((m \pi / a) y^{\prime}\right)}{\beta_{m}} \\
& \times \mathrm{e}^{-\jmath \beta_{m}\left|x-x^{\prime}\right|}
\end{aligned}
$$

where $\beta_{m}=\sqrt{k^{2}-((m \pi / a))^{2}}$

In this case, the electric field has only $z$ component, so the total field must vanish at the contour of the scatterers

$$
\left.E_{z}^{\mathrm{s}}\right|_{T}+\left.E_{z}^{\mathrm{i}}\right|_{T}=0
$$

which leads to

$$
E_{z}^{\mathrm{i}}(x, y)=-\jmath k \eta \int_{T} J_{z}\left(x^{\prime}, y^{\prime}\right) G\left(x, y, x^{\prime}, y^{\prime}\right) \mathrm{d} t^{\prime}
$$

where $(x, y)$ is any point along the contour of the scatterers.

\section{MoM discretisation}

To discretise (6) using MoM, pulses are used as basis functions and deltas as test functions, which is known as point matching [3].

The contour of the $2 \mathrm{D}$ scatterers is split into a finite set of cells, $c_{n} n=1,2, \ldots, N$. Each basis function $n$ is a pulse that will be non-zero only inside the $n$th cell

$$
p_{n}(t)= \begin{cases}1, & \text { if } t \in c_{n} \\ 0, & \text { elsewhere }\end{cases}
$$

The current is approximated by a linear combination of the basis functions

$$
J_{z}(x, y)=J_{z}(x(t), y(t))=J_{z}(t) \simeq \sum_{n=1}^{N} j_{n} p_{n}(t)
$$

Substituting in (6)

$$
E_{z}^{\mathrm{i}}(x, y)=-\jmath k \eta \sum_{n=1}^{N} j_{n} \int_{c_{n}} G\left(x, y, x^{\prime}, y^{\prime}\right) \mathrm{d} t^{\prime}
$$

Using $N$ delta functions placed at the centre of each cell as test functions, we obtain

$$
E_{z}^{\mathrm{i}}\left(x_{m}, y_{m}\right)=\sum_{n=1}^{N} j_{n}\left[-\jmath k \eta \int_{c_{n}} G\left(x_{m}, y_{m}, x^{\prime}, y^{\prime}\right) \mathrm{d} t^{\prime}\right]
$$


where $\left(x_{m}, y_{m}\right)$ is the centre of the $m$ th cell. In matrix form we have

$$
E_{z}^{\mathrm{i}}\left(x_{m}, y_{m}\right)=\sum_{n=1}^{N} Z_{m n} j_{n}, \quad m \in[1, \ldots, N]
$$

where

$$
Z_{m n}=-\jmath k \eta \int_{c_{n}} G\left(x_{m}, y_{m}, x^{\prime}, y^{\prime}\right) \mathrm{d} t^{\prime}
$$

In order to compute the elements $Z_{m n}$, we will treat all cells as if they were strips, with a straight contour. This will speed up the calculations, and not much error will be introduced because all cells will be electrically very small. Using this approximation, we obtain

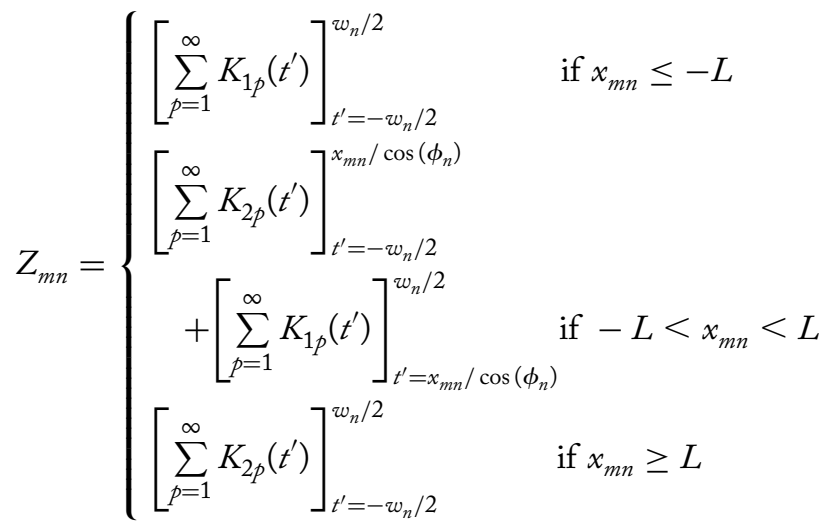

where

$$
\begin{aligned}
K_{1 p}\left(t^{\prime}\right)= & \frac{k \eta}{a} \cdot \frac{\sin \left((p \pi / a) y_{m}\right)}{\beta_{p}} \\
& \cdot \frac{\left\{k_{1} \cos (\theta)-k_{2} \sin (\theta)\right\} \mathrm{e}^{k_{2} t^{\prime}}}{-\left(k_{1}^{2}+k_{2}^{2}\right)} \mathrm{e}^{j \beta_{p} x_{m n}} \\
K_{2 p}\left(t^{\prime}\right)= & \frac{k \eta}{a} \cdot \frac{\sin \left((p \pi / a) y_{m}\right)}{\beta_{p}} \\
& \cdot \frac{\left\{k_{1} \cos (\theta)+k_{2} \sin (\theta)\right\} \mathrm{e}^{-k_{2} t^{\prime}}}{-\left(k_{1}^{2}+k_{2}^{2}\right)} \mathrm{e}^{-j \beta_{p} x_{m n}}
\end{aligned}
$$

and

$$
\begin{aligned}
L & =\frac{w_{n}}{2} \cos \left(\phi_{n}\right) \\
\theta & =k_{1} t^{\prime}+k_{3} \\
x_{m n} & =x_{m}-x_{n} \\
k_{1} & =\frac{p \pi}{a} \sin \left(\phi_{n}\right) \\
k_{2} & =-j \beta_{p} \cos \left(\phi_{n}\right) \\
k_{3} & =\frac{p \pi}{a} y_{n}
\end{aligned}
$$

In the above expressions, $\left(x_{n}, y_{n}\right), w_{n}$ and $\phi_{n}$ mean, respectively, the centre position, length and angle with the $x$-axis of the $n$th cell.
The main advantage of this new method is that the series in (13) converge faster than the series of the original Green's function as in (4).

\section{Computation of scattering parameters}

Solving the matrix system defined by (11), we obtain the amplitudes $j_{n}$ of the basis functions that approximate the current along the surface of the scatterers. This information must be used to compute the scattering parameters that characterise the guided structure.

Since only $T E_{p 0}$ modes are excited in the structure, the electric field can be expanded in terms of these modes according to the following expression [20]

$$
\begin{aligned}
E_{z}(x, y)= & \sum_{p=1}^{\infty} a_{i}^{p}(x) \sqrt{\frac{2 Z_{0 p}}{a b}} \sin \left(\frac{p \pi}{a} y\right) \\
& +\sum_{p=1}^{\infty} b_{i}^{p}(x) \sqrt{\frac{2 Z_{0 p}}{a b}} \sin \left(\frac{p \pi}{a} y\right)
\end{aligned}
$$

where $i=1$ for the input guide, $i=2$ for the output guide and

$$
\begin{aligned}
a_{1}^{p}(x) & =a_{1}^{p} \mathrm{e}^{-j \beta_{p} x} \\
a_{2}^{p}(x) & =a_{2}^{p} \mathrm{e}^{j \beta_{p} x} \\
b_{1}^{p}(x) & =b_{1}^{p} \mathrm{e}^{j \beta_{p} x} \\
b_{2}^{p}(x) & =b_{2}^{p} \mathrm{e}^{-j \beta_{p} x} \\
Z_{0 p} & =\omega \mu / \beta_{p}
\end{aligned}
$$

The scattering parameters are defined as

$$
S_{i j}^{(p, q)}=\left.\frac{b_{i}^{p}\left(X_{i}\right)}{a_{j}^{q}\left(X_{j}\right)}\right|_{a_{i}^{q}\left(X_{i}\right)=0, \forall q, a_{j}^{p}\left(X_{j}\right)=0, \quad \forall p \neq q}
$$

where $X_{i}(i=[1,2])$ is the $x$ coordinate of the position of the reference planes at the input $(i=1)$ and output $(i=2)$ ports.

Substituting (4) and (8) in (1), we obtain the field scattered by any object placed inside the guide, that is, between the parallel plates

$$
\begin{aligned}
& E_{z}^{\mathrm{s}}\left(X_{2}, y\right)=\sum_{p=1}^{\infty} \sin \left(\frac{p \pi}{a} y\right) \mathrm{e}^{-j \beta_{p} X_{2}} \sum_{n=1}^{N} j_{n} A_{p n}^{+} \mathrm{e}^{j \beta_{p} x_{n}} \\
& E_{z}^{\mathrm{s}}\left(X_{1}, y\right)=\sum_{p=1}^{\infty} \sin \left(\frac{p \pi}{a} y\right) \mathrm{e}^{j \beta_{p} X_{1}} \sum_{n=1}^{N} j_{n} A_{p n}^{-} \mathrm{e}^{-j \beta_{p} x_{n}}
\end{aligned}
$$


where

$$
\begin{aligned}
& A_{p n}^{+}=-\frac{k \eta}{a} \frac{1}{\beta_{p}} \frac{\left[\left\{k_{1} \cos (\theta)+k_{2} \sin (\theta)\right\} \mathrm{e}^{-k_{2} t^{\prime}}\right]_{-w_{n} / 2}^{w_{n} / 2}}{-\left(k_{1}^{2}+k_{2}^{2}\right)} \\
& A_{p n}^{-}=-\frac{k \eta}{a} \frac{1}{\beta_{p}} \frac{\left[\left\{k_{1} \cos (\theta)-k_{2} \sin (\theta)\right\} \mathrm{e}^{k_{2} t^{\prime}}\right]_{-w_{n} / 2}^{w_{n} / 2}}{-\left(k_{1}^{2}+k_{2}^{2}\right)}
\end{aligned}
$$

and $\theta=k_{1} t^{\prime}+k_{3}$.

Comparing (23) and (24) with (16), we can derive the amplitudes of the waves scattered by the object inside the guide at the input and output reference planes

$$
\begin{aligned}
& b_{1}^{p}\left(X_{1}\right)=\mathrm{e}^{j \beta_{p} X_{1}} \sqrt{\frac{a b}{2 Z_{0 p}}} \sum_{n=1}^{N} j_{n} A_{p n}^{-} \mathrm{e}^{-j \beta_{p} x_{n}} \\
& b_{2}^{p}\left(X_{2}\right)=\mathrm{e}^{-j \beta_{p} X_{2}} \sqrt{\frac{a b}{2 Z_{0 p}}} \sum_{n=1}^{N} j_{n} A_{p n}^{+} \mathrm{e}^{j \beta_{p} x_{n}}
\end{aligned}
$$

To compute $S_{11}$ and $S_{21}$, we excite the guide with the following incident field

$$
E_{z}^{\mathrm{i}}(x, y)=\sin \left(\frac{q \pi}{a} y\right) \mathrm{e}^{-\jmath \beta_{q} x}
$$

and use (11) to compute the weights $j_{n}$ that define the current induced over the surface of the metallic object placed inside the guide. These weights are substituted in (27).

Comparing (28) with (16), we derive the value of the incident waves to the object inside the guide at the input and output reference planes

$$
a_{1}^{q}(x)=\sqrt{\frac{a b}{2 Z_{0 q}}} \mathrm{e}^{-j \beta_{q} x} \quad a_{2}^{q}(x)=0
$$

Finally,

$$
S_{11}^{(p, q)}=\frac{b_{1}^{p}\left(X_{1}\right)}{a_{1}^{q}\left(X_{1}\right)}=\frac{\sqrt{Z_{0 q}} \mathrm{e}^{j \beta_{p} X_{1}}}{\sqrt{Z_{0 p}} \mathrm{e}^{-j \beta_{q} X_{1}}} \sum_{n=1}^{N} j_{n} A_{p n}^{-} \mathrm{e}^{-j \beta_{p} x_{n}}
$$

For $S_{21}$, we have to distinguish between two possibilities

$$
\begin{aligned}
& -q=p \\
& \left.S_{21}^{(p, p)}=\frac{b_{2}^{p}\left(X_{2}\right)+a_{1}^{p}\left(X_{2}\right)}{a_{1}^{p}\left(X_{1}\right)}=\frac{\mathrm{e}^{-j \beta_{p} X_{2}}}{\mathrm{e}^{-j \beta_{p} X_{1}}} \sum_{n=1}^{N} j_{n} A_{p n}^{+} \mathrm{e}^{j \beta_{p} x_{n}}+1\right)
\end{aligned}
$$

- $q \neq p$

$$
S_{21}^{(p, q)}=\frac{b_{2}^{p}\left(X_{2}\right)}{a_{1}^{q}\left(X_{1}\right)}=\frac{\sqrt{Z_{0 q}} \mathrm{e}^{-j \beta_{p} X_{2}}}{\sqrt{Z_{0 p}} \mathrm{e}^{-j \beta_{q} X_{1}}} \sum_{n=1}^{N} j_{n} A_{p n}^{+} \mathrm{e}^{j \beta_{p} x_{n}}
$$

To compute $S_{12}$ and $S_{22}$, we change the excitation to the second port

$$
E_{z}^{\mathrm{i}}(x, y)=\sin \left(\frac{q \pi}{a} y\right) \mathrm{e}^{j \beta_{q} x}
$$

and recompute the current weights $j_{n}$. Now, the incident waves are defined as

$$
a_{1}^{q}(x)=0 \quad a_{2}^{q}(x)=\sqrt{\frac{a b}{2 Z_{0 q}}} \mathrm{e}^{j \beta_{q} x}
$$

and

$$
\begin{gathered}
S_{22}^{(p, q)}=\frac{\sqrt{Z_{0 q}} \mathrm{e}^{-j \beta_{p} X_{2}}}{\sqrt{Z_{0 p}} \mathrm{e}^{j \beta_{q} X_{2}}} \sum_{n=1}^{N} j_{n} A_{p n}^{+} \mathrm{e}^{j \beta_{p} x_{n}} \\
\left.S_{12}^{(p, p)}=\frac{\mathrm{e}^{j \beta_{p} X_{1}}}{\mathrm{e}^{j \beta_{p} X_{2}}} \sum_{n=1}^{N} j_{n} A_{p n}^{-} \mathrm{e}^{-j \beta_{p} x_{n}}+1\right) \\
S_{12}^{(p, q)}=\frac{\sqrt{Z_{0 q}} \mathrm{e}^{j \beta_{p} X_{1}}}{\sqrt{Z_{0 p}} \mathrm{e}^{j \beta_{q} X_{2}}} \sum_{n=1}^{N} j_{n} A_{p n}^{-} \mathrm{e}^{-j \beta_{p} x_{n}}
\end{gathered}
$$

\section{Convergence analysis}

The computation of the $Z_{m n}$ elements of (13) involves the summation of an infinite series over the index $p$. This series must be truncated, and only a finite number of terms $P$ can be considered in the computer.

Following the same idea in [14-17], we can define an auxiliary series that allows us to accelerate the summation of (13). In order to do that, we substitute $\beta_{p}$ and $k_{1}^{2}+k_{2}^{2}$ by asymptotically equivalent expressions

$$
\begin{aligned}
& \beta_{p}=-j \sqrt{\left(\frac{p \pi}{a}\right)^{2}-k^{2}} \longleftrightarrow-j \frac{p \pi}{a} \\
& k_{1}^{2}+k_{2}^{2}=\left(\frac{p \pi}{a}\right)^{2}-k^{2} \cos ^{2} \phi \longleftrightarrow\left(\frac{p \pi}{a}\right)^{2}-\left(\frac{\pi}{a}\right)^{2} p
\end{aligned}
$$

The original and the equivalent expressions converge when $p \rightarrow \infty$. 
Substituting $\beta_{p}$ and $k_{1}^{2}+k^{2}$ in (14) by their asymptotically equivalent expressions we obtain two new series

$$
\begin{aligned}
K_{1}^{\prime}\left(t^{\prime}\right)= & \sum_{p=2}^{\infty} \frac{k \eta}{a} \frac{\sin \left((p \pi / a) y_{m}\right)}{-J(p \pi / a)} \frac{\left\{k_{1} \cos (\theta)-k_{2}^{\prime} \sin (\theta)\right\} \mathrm{e}^{k_{2}^{\prime} t^{\prime}}}{-\left(((p \pi / a))^{2}-(\pi / a)^{2} p\right)} \\
& e^{(p \pi / a) x_{m n}}=\sum_{p=2}^{\infty} K_{1 p}^{\prime}\left(t^{\prime}\right) \\
K_{2}^{\prime}\left(t^{\prime}\right)= & \sum_{p=2}^{\infty} \frac{k \eta}{a} \frac{\sin \left((p \pi / a) y_{m}\right)}{-J(p \pi / a)} \frac{\left\{k_{1} \cos (\theta)+k_{2}^{\prime} \sin (\theta)\right\} \mathrm{e}^{-k_{2}^{\prime} t^{\prime}}}{-\left(((p \pi / a))^{2}-(\pi / a)^{2} p\right)} \\
& \mathrm{e}^{-(p \pi / a) x_{m n}}=\sum_{p=2}^{\infty} K_{2 p}^{\prime}\left(t^{\prime}\right)
\end{aligned}
$$

where $k_{2}^{\prime}=-(p \pi / a) \cos \left(\phi_{n}\right)$.

Using the following expression

$$
\sum_{p=2}^{\infty} \frac{\varepsilon^{p}}{p(p-1)}=(1-\varepsilon) \log (1-\varepsilon)+\varepsilon \quad \text { if }|\varepsilon| \leq 1
$$

the series in (39) and (40) can be summed analytically

$$
\begin{aligned}
K_{1}^{\prime}\left(t^{\prime}\right)= & \frac{k \eta}{a} \frac{j}{2}\left(\frac{a}{\pi}\right)^{2}\left[\sin \phi_{n} \operatorname{Im}\left(M_{1}\left(t^{\prime}\right)+M_{2}\left(t^{\prime}\right)\right)\right. \\
& \left.-\cos \phi_{n} \operatorname{Re}\left(M_{1}\left(t^{\prime}\right)-M_{2}\left(t^{\prime}\right)\right)\right] \\
K_{2}^{\prime}\left(t^{\prime}\right)= & \frac{k \eta}{a} \frac{j}{2}\left(\frac{a}{\pi}\right)^{2}\left[\sin \phi_{n} \operatorname{Im}\left(M_{3}\left(t^{\prime}\right)+M_{4}\left(t^{\prime}\right)\right)\right. \\
& \left.+\cos \phi_{n} \operatorname{Re}\left(M_{3}\left(t^{\prime}\right)-M_{4}\left(t^{\prime}\right)\right)\right]
\end{aligned}
$$

where

$$
\begin{gathered}
M_{i}\left(t^{\prime}\right)=\left(1-m_{i}\left(t^{\prime}\right)\right) \log \left(1-m_{i}\left(t^{\prime}\right)\right)+m_{i}\left(t^{\prime}\right), i \in[1,2,3,4] \\
m_{1}\left(t^{\prime}\right)=\mathrm{e}^{\pi / a\left[J\left(y_{m}+y_{n}+t^{\prime} \sin \phi_{n}\right)+\left(x_{m}-x_{n}-t^{\prime} \cos \phi_{n}\right)\right]} \\
m_{2}\left(t^{\prime}\right)=\mathrm{e}^{\pi / a\left[J\left(y_{m}-y_{n}-t^{\prime} \sin \phi_{n}\right)+\left(x_{m}-x_{n}-t^{\prime} \cos \phi_{n}\right)\right]} \\
m_{3}\left(t^{\prime}\right)=\mathrm{e}^{\pi / a\left[J\left(y_{m}+y_{n}+t^{\prime} \sin \phi_{n}\right)-\left(x_{m}-x_{n}-t^{\prime} \cos \phi_{n}\right)\right]} \\
m_{4}\left(t^{\prime}\right)=\mathrm{e}^{\pi / a\left[J\left(y_{m}-y_{n}-t^{\prime} \sin \phi_{n}\right)-\left(x_{m}-x_{n}-t^{\prime} \cos \phi_{n}\right)\right]}
\end{gathered}
$$

Using the auxiliary series previously defined we can reformulate the series of (13) and give raise to a new expression that converges faster

$$
\begin{gathered}
\sum_{p=1}^{\infty} K_{i p}\left(t^{\prime}\right)=K_{i 1}\left(t^{\prime}\right)+\sum_{p=2}^{\infty}\left\{K_{i p}\left(t^{\prime}\right)-K_{i p}^{\prime}\left(t^{\prime}\right)\right\}+K_{i}^{\prime}\left(t^{\prime}\right), \\
i \in[1,2]
\end{gathered}
$$

The convergence ratio for the series of (45) in the worst case (when $x_{m n}=0$ and $Z_{m n}$ represents the interaction between cells not separated along the propagation direction) is

$$
\left[\frac{p}{\sqrt{p^{2}-(k a / \pi)^{2}}\left[p^{2}-(k a \cos \phi / \pi)^{2}\right]}-\frac{1}{p(p-1)}\right]
$$

This convergence ratio is greater than that in the method presented in [17], where the convergence ratio is

$$
\left[\frac{1}{\sqrt{p^{2}-(k a / \pi)^{2}}}-\frac{1}{p}\right]
$$

The decreasing term of (46) tends to zero faster than the term of (47) [17], as shown in Fig. 2.

In addition it is necessary to consider that in [17] the summation of the series provides with the evaluation of the PPW Green's function, which still must be integrated to obtain the $Z_{m n}$ elements. The authors in [17] propose a numerical integration of the summed Green's function, which means that the Green's function needs to be evaluated (and the series summed) several times according to the chosen quadrature rule. In this paper we have integrated analytically the terms of the series prior to its summation. Thus, once the faster-convergent series has been summed, we have already obtained the $Z_{m n}$ elements, which implies an additional gain in efficiency when compared with the method in [17].

A convergence analysis for the corrected series of (45) must be performed in order to fix the optimum value of $P$, the maximum value of $p$ that we use to truncate the series. For that purpose, we must first note that this optimum number $P$ strongly depends on the separation $x_{m n}=x_{m}-x_{n}$ along the propagation direction ( $x$ axis) between cells $m$ and $n$. When the cells are far away along $x$, we need very few terms of the series to accurately evaluate it since the exponential terms in (14), (39) and (40) vanish very quickly as $p$ increases. In consequence, two different values for $P$ must be sought. One for the case when $x_{m n}=0$ (worst case), and another for the case when $x_{m n} \neq 0$. We have

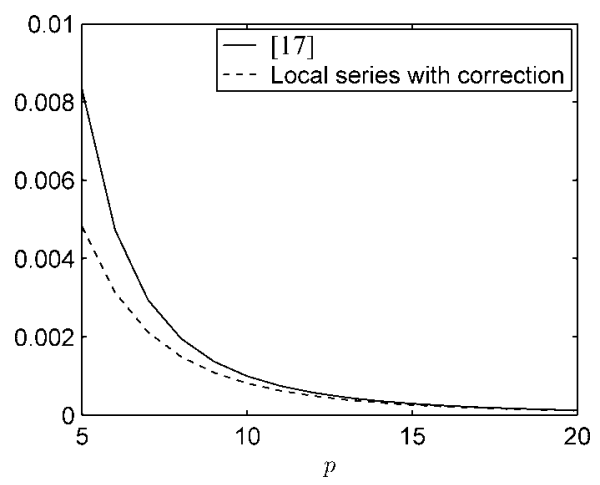

Figure 2 Convergence of the corrected series used in the computation of the elements of $\overline{\bar{Z}}$ in [17] and in this work 
called $P_{\max }$ to the maximum value of $p$ used to truncate the series when $x_{m n}=0$. When $x_{m n} \neq 0$, we have decided to truncate the series when the modulus of the exponential $\mathrm{e}^{-\jmath \beta_{p}\left|x_{m n}\right|}$ decays below a threshold value $\eta$ or $p$ reaches $P_{\max }$, whichever occurs first.

In order to fix $P_{\max }$, Fig. 3 shows the convergence of the reflection coefficient of the filter of Fig. 14 as a function of $P_{\max }$. For these calculations $\eta$ has been fixed to $10^{-3}$. Results are shown for different densities of MoM basis functions per wavelength $\left(N_{d}\right)$. These results show a great stability and a smooth convergence behaviour when $P_{\max }$ varies. We have chosen $P_{\max }=50$, which ensures very good accuracy in the results.

Fig. 4 studies the influence of the threshold value $\eta$ in the convergence of the method. As shown in the figure, with $\eta=10^{-3}$ the method has already converged, and this value has been used for all calculations.

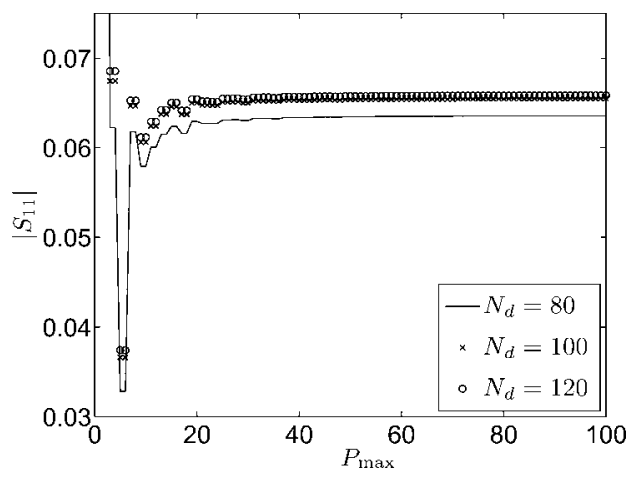

Figure 3 Convergence of $S_{11}$ with the number of modes $P_{\text {max }}$ used to truncate the series of (45) when $x_{m n}=0$

Results are shown for various densities of MoM basis functions $\left(N_{d}\right)$

Results taken from the analysis of the filter of Fig. 12 at $f=11 \mathrm{GHz}\left(\eta=10^{-3}\right)$

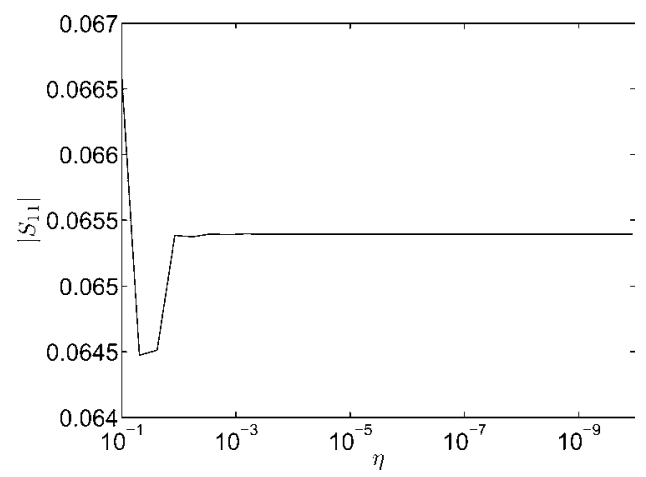

Figure 4 Convergence of $S_{11}$ with $\eta$, the threshold value used to truncate the series of (45) when $x_{m n} \neq 0$

Results taken from the analysis of the filter of Fig. 12 at $f=11 \mathrm{GHz}$

$P_{\max }=50$ and $N_{d}=100$

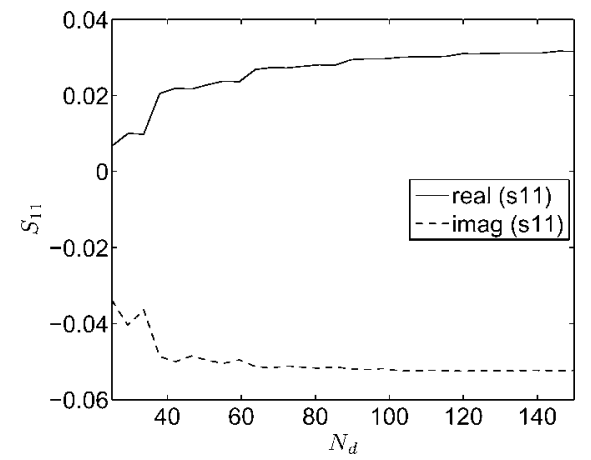

Figure 5 Convergence of $S_{11}$ with $N_{d}$, the density of MoM basis functions per wavelength

Results taken from the analysis of the filter of Fig. 12 at $f=11 \mathrm{GHz}\left(P_{\max }=50\right.$ and $\left.\eta=10^{-3}\right)$

Finally, the convergence of the method with the density of MoM basis functions per wavelength is analysed in Fig. 5. With $N_{d}=100$, the results are stable, and this value has been used in all calculations. This value is valid for straight segments of the contour of the scatterer. However, the rounded segments are approximated by small straight cells, which make necessary a higher density of cells, depending on the radius of the curvature of these rounded segments. Fig. 6 analyses the convergence of the method with the number of straight cell per radian $\Omega$. All calculations have been made with a density of $\Omega>24 / \pi$ cells per radian for the rounded segments of the scatterer contour.

\section{Results}

The efficiency and accuracy of this new method are tested with the analysis of several $H$-plane structures in rectangular waveguide. An Intel(囚) Pentium(®) $4 \mathrm{CPU}$ of $3.00 \mathrm{GHz}$ and $1 \mathrm{~GB}$ of RAM has been used for all the calculations.

The analysis of a centred and an off-centred circular metallic post is presented in Figs. 7 and 8. The equivalent circuital parameters $X_{a}$ and $X_{b}$ of the obstacle (see

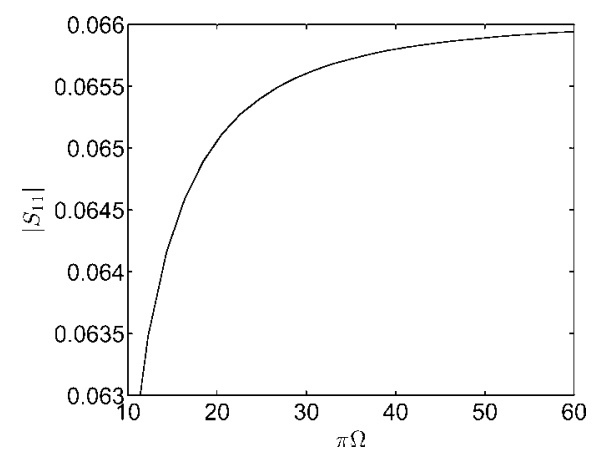

Figure 6 Convergence of $S_{11}$ with $\Omega$, the density of MoM basis functions per radian in curved segments

Results taken from the analysis of the filter of Fig. 14 at $f=11 \mathrm{GHz}\left(P_{\max }=50, \eta=10^{-3}\right.$ and $\left.N_{d}=100\right)$ 


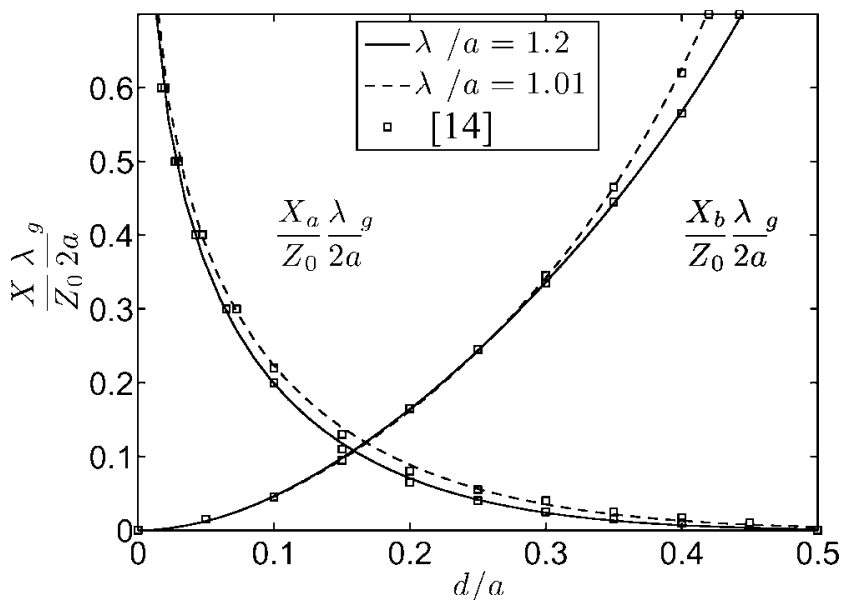

Figure 7 Circuital parameters of a centred metallic cylinder of diameter $d\left(P_{\max }=50, \eta=10^{-3}, N_{d}=100\right.$ and $\Omega=24 \pi)$

definition in [20]) computed with the new method are compared with the results in [14]. The off-centred cylinder is placed at $x=0.3 a$, where $a$ is the waveguide width. A good agreement between both results can be observed. The CPU time required by the new method was only $0.1 \mathrm{~s}$ per point.

The new method has also been used for computing the equivalent inductance of an iris and a window centred inside the waveguide, as shown in Fig. 9. The results have been successfully compared with [20] in Figs. 10 and 11. The CPU time required by the new method was $0.01 \mathrm{~s}$ per point for the iris, and $0.02 \mathrm{~s}$ for the window.

The new method has also been tested with more complex waveguide structures. First, it has been used to analyse a

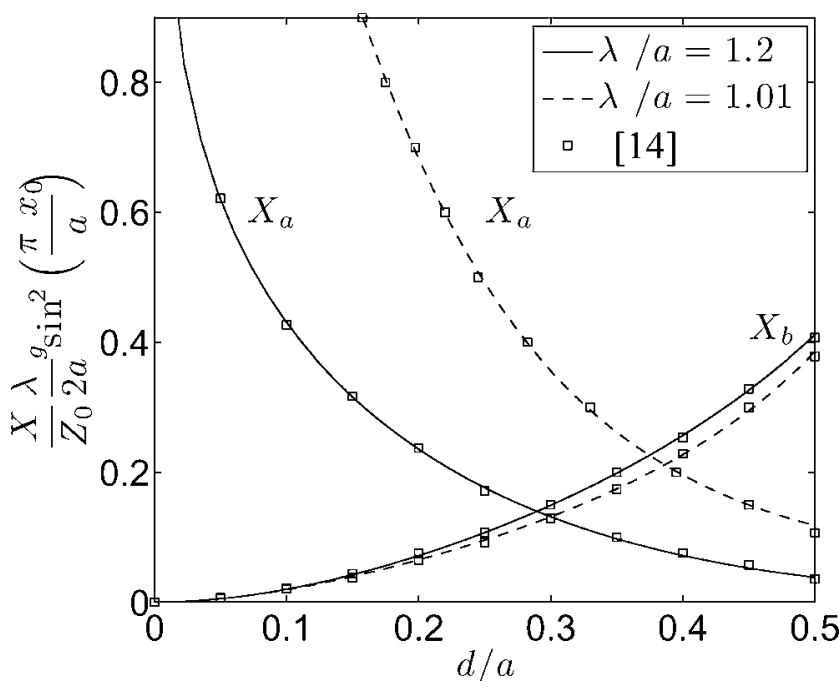

Figure 8 Circuital parameters of an off-centred metallic cylinder of diameter $d\left(P_{\max }=50, \eta=10^{-3}, N_{d}=100\right.$ and $\Omega=24 \pi$ )

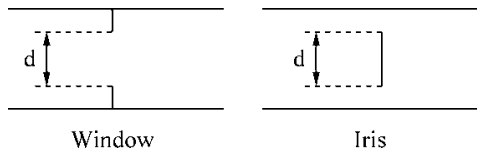

Figure 9 Iris and window analysed with the new method

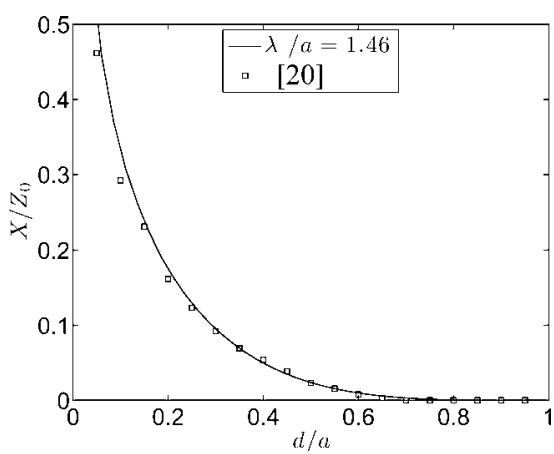

Figure 10 Equivalent inductance of the centred metallic iris $\left(N_{d}=100, P_{\max }=50, \eta=10^{-3}\right)$

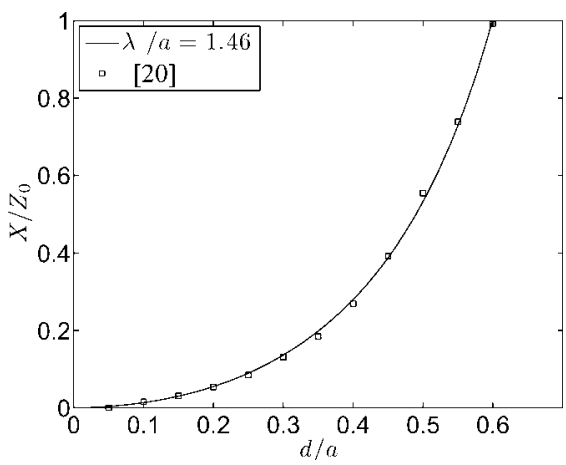

Figure 11 Equivalent inductance of the centred window $\left(N_{d}=100, P_{\text {max }}=50, \eta=10^{-3}\right)$

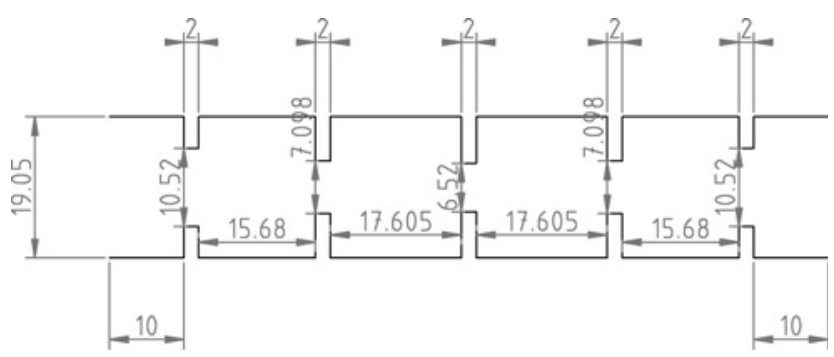

Figure 12 Filter with resonator cavities

All dimensions are in millimetres

standard $\mathrm{H}$-plane waveguide filter with four rectangular resonator cavities. A top view of the filter with all the dimensions is presented in Fig. 12. The filter electrical response is centred at $11 \mathrm{GHz}$, and the bandwidth is $300 \mathrm{MHz}$. 


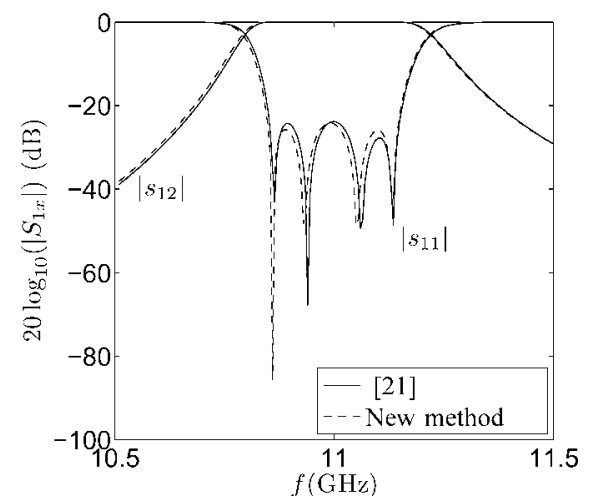

Figure 13 Scattering parameters for the four-cavity filter $\left(N_{d}=100, P_{\max }=50, \eta=10^{-3}\right)$

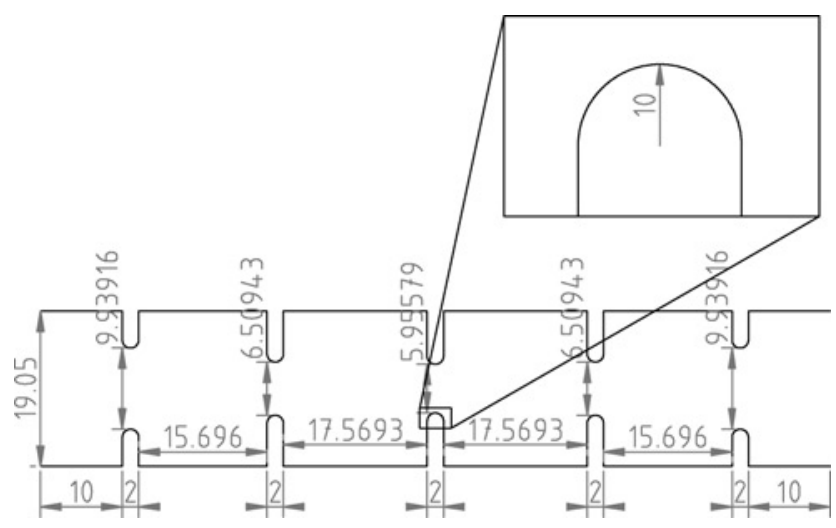

Figure 14 Filter with rounded corners $(r=1 \mathrm{~mm})$

All dimensions are in millimetres

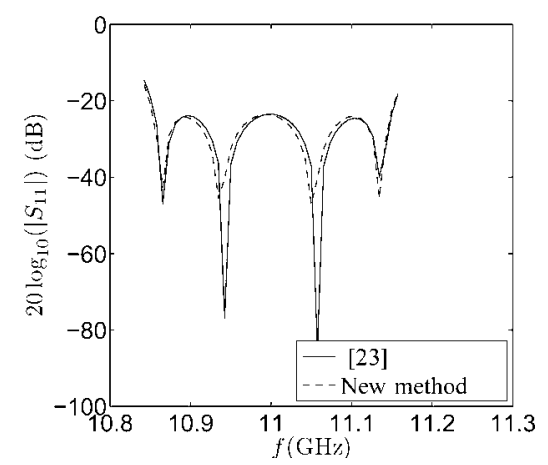

Figure 15 Scattering parameters for the four-cavity filter with rounded corners $(r=1 \mathrm{~mm})\left(N_{d}=100, P_{\max }=50\right.$, $\eta=10^{-3}, \Omega=24 \pi$ )

The scattering parameters of the filter are presented in Fig. 13. A good agreement with the results of [21] can be observed. The CPU time was $0.55 \mathrm{~s}$ per frequency point.

Finally, the accuracy and efficiency of the new method are tested with the analysis of an inductive filter with four rectangular resonator cavities, but considering rounded corners in the coupling windows. These rounded corners appear due to the mechanisation of these filters using milling and die-casting techniques [22]. The top view of the filter with all the dimensions is shown in Fig. 14. The radius of the rounded corners is $1 \mathrm{~mm}$.

Fig. 15 compares the scattering parameters of the filter with rounded corners with the results from [23]. A good agreement between both curves can be observed. The CPU time required was only $0.94 \mathrm{~s}$ per frequency point.

\section{Conclusions}

A new method for the full-wave analysis of arbitrarily shaped $\mathrm{H}$-plane two-port structures in rectangular waveguide technology is presented. The method is based on the use of the Green's function of an infinite line source placed inside two parallel plates, which is expressed as the summation of an infinite series of guided modes. The new method presented here improves the numerical efficiency of previous classical techniques, which were based on the direct summation of the series related to the cited Green's function. Instead, we propose to integrate the terms of such series within the frame of the MoM, thus resulting in new series expressions which converges faster.

An exhaustive analysis of the convergence of the new method proposed has been performed, and optimal values for the parameters of the method have been found and used in all calculations, thus ensuring the accuracy of the results in a very reduced computational effort. Then, the new method has been validated with the analysis of some simple canonical structures. Finally, it has been successfully applied to the efficient analysis of other more complex structures, such as a four-cavity rectangular waveguide filter with rounded corners in the coupling windows.

\section{References}

[1] PETERSON A.F., RAY S.L., MITTRA R.: 'Computational methods for electromagnetics', IEEE Press/OUP Series on Electromagnetic Wave Theory, (IEEE Press/OUP, 1997)

[2] CHEW W.C., JIN J.-M., MICHIELSSEN E., SONG J.: 'Fast and efficient algorithms in computational electromagnetics' (Artech House, Norwood, MA, 2001)

[3] HARRINGTON R.F.: 'Field computation by moment methods', IEEE Press/OUP Series on Electromagnetic Wave Theory (IEEE Press/OUP, 1993)

[4] GUGLIELMI M., NEWPORT C.: 'Rigorous, multimode equivalent network representation of inductive discontinuities', IEEE Trans. Microw. Theory Tech., 1990, 38, (11), pp. 1651-1659 
[5] ROZZI T., MOGLIE F., MORINI A., GULLOCH W., POLITI M.: 'Accurate full-band equivalent circuits of inductive posts in rectangular waveguide', IEEE Trans. Microw. Theory Tech., 1992, 40, (5), pp. 1000-1009

[6] CONCIAURO G., ARCIONI P., BRESSAN M., PERREgRINI L.: 'Wideband modeling of arbitrarily shaped H-plane waveguide components by the BI-RME method', IEEE Trans. Microw. Theory Tech., 1996, 44, (7), pp. 1057-1066

[7] GUGLIELMI M.: 'Simple CAD procedure for microwave filters and multiplexers', IEEE Trans. Microw. Theory Tech., 1994, 42, (7), pp. 1347-1352

[8] ARNDT F., BEYER R., REITER J.M., SIEVERDING T., WOLF T.: 'Automated design of waveguide components using hybrid mode-matching/numerical EM building-blocks in optimization-oriented CAD frameworks-State-of-the-art and recent advances', IEEE Trans. Microw. Theory Tech., 1997, 45, (5), pp. 747-760

[9] YIN S., VASILYEVA T., PRAMANICK P.: 'Use of three-dimensional field simulators in the synthesis of waveguide round rod bandpass filters', Int. J. RF Microw. Comput. Aided Eng., 1998, 8, (6), pp. 484-497

[10] MACPHIE R.H., WU K.-L.: 'A full wave modal analysis of arbitrarily shaped waveguide discontinuities using the finite plane-wave series expansion', IEEE Trans. Microw. Theory Tech., 1999, 47, (2), pp. 232-237

[11] ESTEBAN H., COGOLLOS S., BORIA V.E., SAN BLAS A.A., FERRANDO M.: 'A new hybrid mode-matching/numerical method for the analysis of arbitrarily shaped inductive obstacles and discontinuities in rectangular waveguides', IEEE Trans. Microw. Theory Tech., 2002, 50, (4), pp. 1219-1224

[12] TAl C.-T.: 'Dyadic green functions in electromagnetic theory' (IEEE Press, Piscataway, NJ, 1993)

[13] COLLIN R.E.: 'Field theory of guided waves' (IEEE Press, New York, 1991, 2nd edn.)
[14] LEVIATAN Y., LI P.G., ADAMS A.T., PERINI J.: 'Single post inductive obstacle in rectangular waveguide', IEEE Trans. Microw. Theory Tech., 1983, 31, (10), pp. 806-812

[15] LEVIATAN Y., SHAU D.-H., ADAMS A.T.: 'Numerical study of the current distribution on a post in a rectangular waveguide', IEEE Trans. Microw. Theory Tech., 1984, 32, (10), pp. $1411-1415$

[16] LI P.G., AdAMS A.T., LEVIATAN Y., PERINI J.: 'Multiple post inductive obstacles in rectangular waveguide', IEEE Trans. Microw. Theory Tech., 1984, 32, (4), pp. 365-373

[17] AUDA H., HARRINGTON R.: 'Inductive posts and diaphragms of arbitrary shape and number in a rectangular waveguide', IEEE Trans. Microw. Theory Tech., 1984, 32, (6), pp. 606-613

[18] BALANIS C.A.: 'Advanced engineering electromagnetics' (John Wiley \& Sons, Inc, 1989)

[19] DUFFY D.G.: 'Green's functions with applications' (Chapman \& Hall/CRC, 2001)

[20] MARCUVITZ N.: 'Waveguide handbook' (IEE Electromagnetic Wave Series 21, London, 1986)

[21] BORIA V.E.: 'Analisis de problemas electromagneticos mediante metodos modales y matrices generalizadas, y aplicaciones', Ph.D. dissertation, Universidad Politecnica de Valencia, Valencia, 1997

[22] MORRO J.V., eSteBAN H., BACHILleR C., BORIA V.e.: 'Automated design of complex waveguide filters for space systems: a case study', Int. J. RF Microw. Comput. Aided Eng., 2007, 17, (1), pp. 84-89

[23] MORRO J.V., ESTEBAN H., BORIA V.E., BACHILLER C., COGOLLOS S., COVES A.: 'Efficient automated design of $\mathrm{H}$-plane filters with rounded corners using ASM with a segmentation strategy and hybrid optimization techniques'. 2004 IEEE Antennas and Propagation Society Int. Symp. Digest, Monterey, California, June 2004, vol. 3, pp. 2735-2738 